\title{
Nuevos métodos de diagnóstico en Periodoncia. Métodos bioquímicos
}

\author{
FARIA $\mathbf{R} *$ \\ BELÉN A * \\ BASCONES $\boldsymbol{A} * *$
}

Faria R, Belén A, Bascones A. Nuevos métodos de diagnóstico en Periodoncia. Métodos bioquímicos. Av Periodon Implantol. $2001 ; 13,1: 29-37$

\section{RESUMEN}

Los métodos de diagnóstico tradicionales no son suficientemente fiables y solo nos permiten un diagnóstico retrospectivo sobre la pérdida del nivel de inserción. Luego, con el objetivo de aumentar su fiabilidad han sido desarrollados métodos complementarios de diagnóstico y alternativos para detectar la actividad de la enfermedad periodontal.

En realidad existen un conjunto de nuevos métodos de diagnóstico, basados en la respuesta inmunológica que nos ayudan a hacer un diagnóstico más preciso y eficaz. Es pues, necesario conocer sus ventajas y desventajas y su aplicabilidad clínica.

Fue hecha una revisión de artículos de los últimos dos años del Journal Clinical of Periodontology y en Journal of Periodontol.

\section{PALABRAS CLAVE}

Enfermedad periodontal, indicadores microbiológicos, inflamatorios e inmunológicos, encimas proteolíticas e hidrolíticas.

\section{INTRODUCCIÓN}

La capacidad para diagnosticar la existencia de periodontitis en una localización periodontal, y diferenciarla, por tanto, de una localización sana, ha sido uno de los aspectos más discutidos en los últimos años (1), (2). El conocimiento cada vez más preciso de la naturaleza, etiologia y patogenia de esta enfermedad sigue suscitando una continua busqueda de nuevas estrategias de diagnóstico, y de como adaptarlas a la práctica clínica diaria.

Durante años, el diagnóstico de la Enfermedad Periodontal (EP) se basaba en examenes clínicos y radiográficos, que evidenciaban la pérdida de inserción y de soporte óseo. No obstante, la determinación de la profundidad de sondaje, pérdida de inserción y nivel de la cresta ósea sólo permite realizar un diagnóstico retrospectivo de la pérdida de soporte periodontal. Asimismo, durante decadas se pensó que la enfermedad periodontal, una vez iniciada, evolucionaba de una forma lenta y continua. Este concepto de progresión lineal de la enfermedad arranca de los estudios de Russell et al. (3), que mostraban una mayor destrucción de soporte en los pacientes de edad más avanzada. Igualmente, se consideraba que, en presencia de placa bacteriana, y una vez iniciada la enfermedad, esta evolucionaría de una forma similar en los diferentes individuos no tratados. Años después, estudios realizados en grandes grupos de población ajenos a cualquier tipo de cuidados dentales, permiten postular un nuevo modelo de progresión de la periodontitis, que considera como determi-

* Alumno del Master de Periodoncia de la Universidad Complutense de Madrid.

** Catedrático en Medicina Bucal y Periodoncia de la UCM. 
nante el factor de la susceptibilidad individual frente a la agresión bacteriana (4). Según este nuevo modelo, dentro de un mismo grupo de individuos sometidos a las mismas condiciones ambientales, y con nivel socioeconómico y hábitos higiénicos similares, se pueden encontrar distintos patrones de evolución de la enfermedad periodontal. Incluso en los pacientes tratados, se econtró que, a pesar de la terapia de mantenimiento, podrían existir períodos o brotes de exacerbación de la enfermedad. Estos brotes de pérdida de inserción, que inicialmente se consideraban no justificados, se relacionaron con alteraciones de la flora bacteriana o de las defensas del propio huesped (5). Así, la presencia, por ejemplo, de Actynobacillus actynomycetemcomitans ( $\mathrm{Aa}$ ) se demostró fuertemente asociada a localizaciones enfermas, mientras que la de Streptococos sanguis lo estaba a las localizaciones sanas.

En las últimas decadas, se han tratado de desarrollar nuevas lineas de estudio y de diagnóstico basadas en el estudio de mediadores inmunológicos y bioquímicos y de su relación con la EP, como resultado de la respuesta individual a la enfermedad. Estos mediadores incluyen algunos considerados especificos de la respuesta anti-infecciosa, como son los anticuerpos frente a determinados patógenos, así como otros que representan una reacción negativa del huésped. En este último caso se incluyen aquellas sustancias derivadas de la respuesta inflamatoria específica, que tienen un efecto lesivo para el propio huésped, como son algunas enzimas y citoquinas liberadas por las células inflamatorias. La respuesta del huésped a la infección periodontal puede relacionarse con un proceso de infección aguda, de respuesta humoral, o de respuesta celular. Cada una de estas formas puede ser valorada mediante pruebas diagnósticas para la detección de los mediadores relacionados con ellas.

\section{RECOGIDA DE MUESTRAS A PARTIR DEL FLUIDO CREVICULAR}

El fluido crevicular es un exudado que contiene enzimas, células descamadas, restos celulares y otros productos derivados del epitelio sulcular. La importancia del fluido crevicular en el diagnóstico periodontal radica en la posibilidad de determinar la presencia de diversas enzimas, marcadores de la inflamación, y restos celulares del huesped cuya presencia podría asociarse con distintos grados de periodontitis. Al igual que el fluido crevicular, la saliva puede ser utilizada, según algunos autores, como medio para la detección de estos marcadores.

Para su análisis, el fluido crevicular debe ser recogi- do de forma que se produzca una minimo deterioro del entorno del surco, y en el menor tiempo posible; como referencia se ha estimado que la recogida no debe durar más de $30 \mathrm{Seg}$ (6). De hecho, la falta de estandarización a la hora de la recojida de las muestras, así como de su análisís, hace difícil establecer comparaciones entre los diferentes estudios publicados, y, por tanto, extraer conclusiones.

Cimasoni et al. en 1983 (6) describieron cuatro métodos posibles para la recogida del fluido, como son las tiras de papel, micropipetas, microjeringas, y tiras de plástico; a estos deben añadirse las técnicas inmunológica y bioquímicas descritas por Rossomando et al. en 1987 (10).

a) Tiras de papel. Las tiras de papel son pequeñas bandas de celulosa que permiten absorber por capilaridad el fluido crevicular cuando son insertadas en el surco gingival, o cuando son colocadas en su entrada. Su principal limitación radica en el hecho de que sólo permiten cuantificar el volumen de fluido generado. Una vez recogidas las muestras, existen varias técnicas para cuantificar el volumen de fluido: mediante análisis colorimétrico, mediante valoración del tamaño de la tira con fluido, y mediante fluorescencia. El método más extendido consiste en la determinación de la penetración de fluido en la tira mediante un dispositivo electrónico (Periotron, ProFlow, Amityville, NY). Las diferencias encontradas son registradas digitalmente para determinar el volumen total de fluido crevicular. La determinación obtenida con esta aparatología puede verse influida por la temperatura ambiental y/o por el grado de humedad; para minimizar esta influencia fue desarrolada una nueva generación de lectores (Periotron 6000, ProFlow, Amityville, NY) considerado por algunos autores como más predecible que su predecesor (7) y (8).

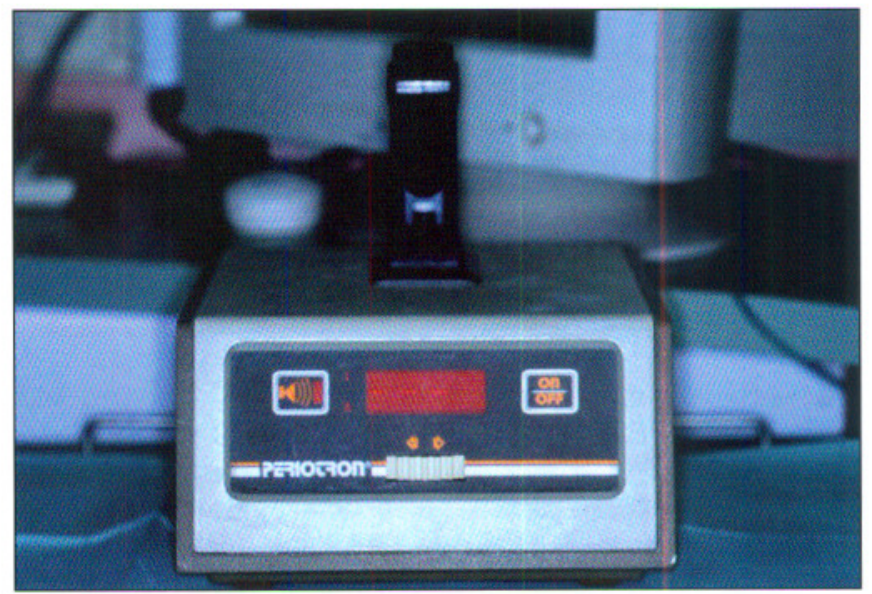

Figura 1. Periotron, ProFlow, Amityville, NY (Periopaper 6) 


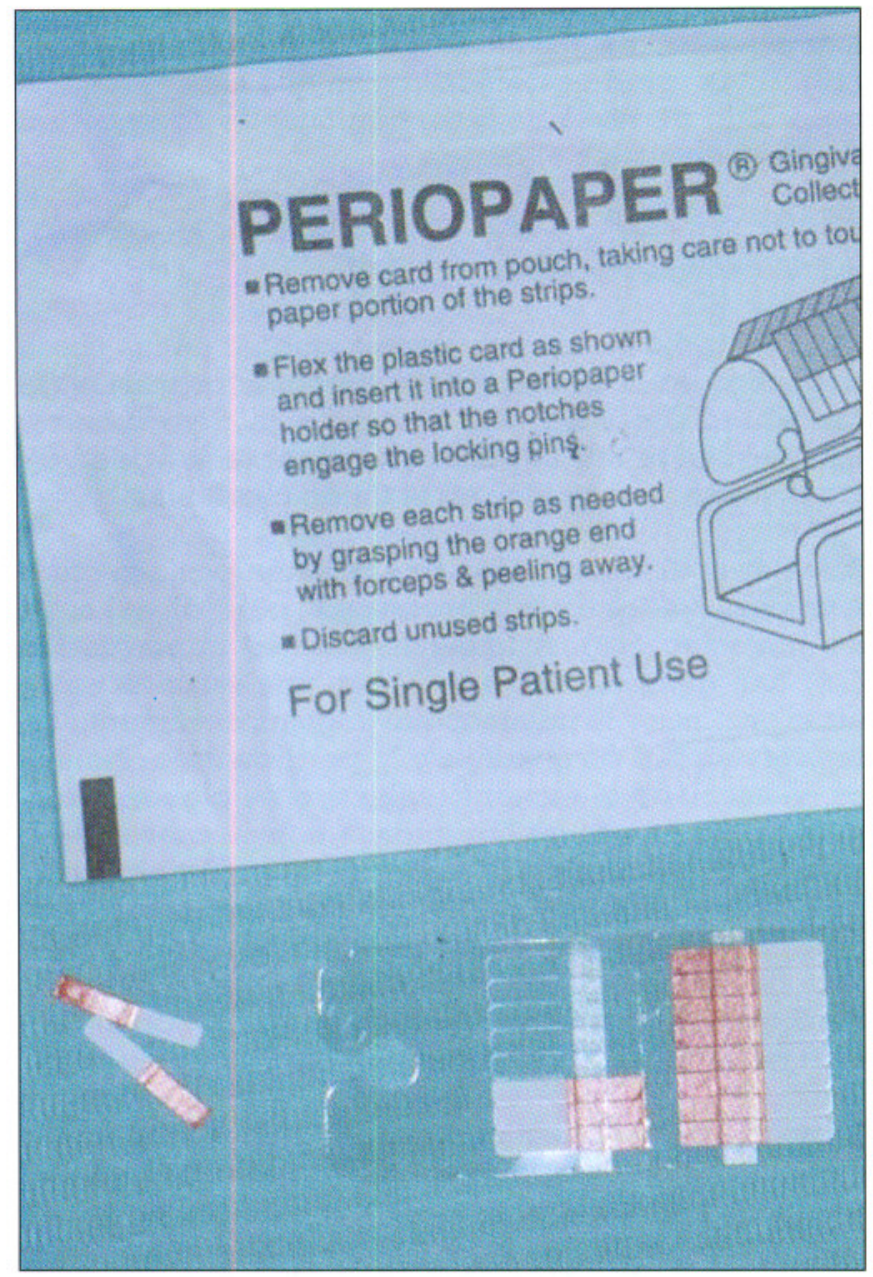

Figura 2. Tiras de periopaper (Periopaper y periopaper 2).

b) Micropipetas. Las micropipetas son dispositivos que permiten recoger pequeños volúmenes de líquido, en este caso fluido gingival, para su cuantificación o análisis cualitativo. Para esto, la pipeta debe ser colocada a la entrada del surco para la recogida del fluido por acción capilar, y medir así el volumen del fluido que entra en el tubo. En relación al análisis del fluido, este método presenta el inconveniente de la alteración en la composición del fluido al entrar en contacto con la superficie del tubo de la pipeta; determinados sustancias podrían quedar adheridas a su superficie, y posteriormente, no podrían ser detectadas.

c) Microjeringas. Este es un metodo de valoración de la cantidad de fluido. Para ello se introduce un volumen conocido de una sustancia tampón mediante una pequeña jeringa en el surco gingival, y posteriormente es arrastrado junto con el fluido previamente existente en el surco. La sustracción de ambos volúmenes nos proporciona el valor correspondiente al fluido crevicular.

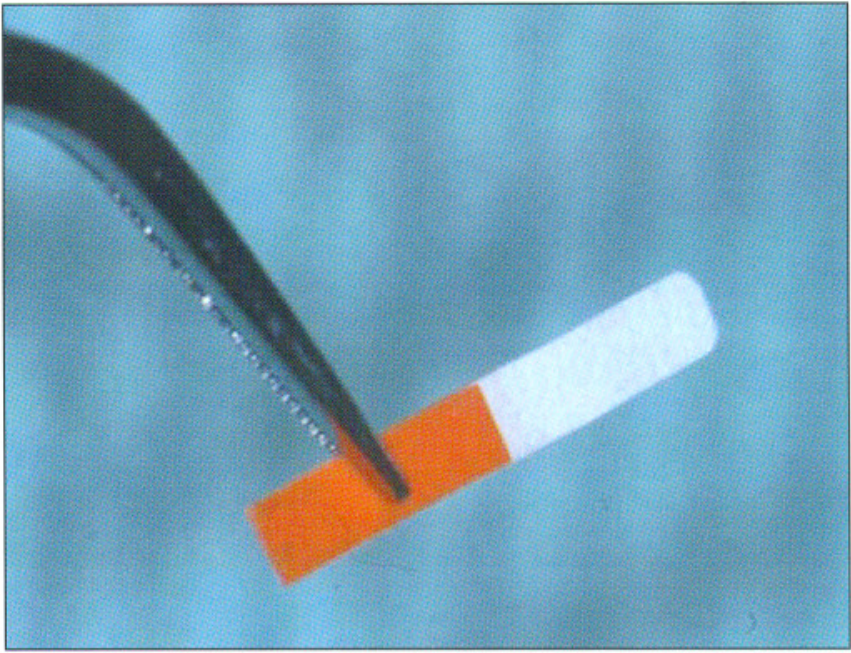

Figura 3. Toma de muestras (Periopaper 3)

d) Tiras de plastico - Estas tiras de plastico sirven para recoja y examinación de leucocitos intracreviculares. Permiten cuantificar el numero de PMN no surco y relacionar su cuantidad con la respuesta inflamatória. (9).

e) Tecnicas imnulogicas y bioquimicas - Técnicas locales que nos permiten, por una unión de un Atc conocido detectar la presencia de una molecula especifica que queremos estudiar. El Atc generalmente con origen animal, es introducido en el surco unido a una pequeña gota magnética. Este complejo es despúes recogido en el surco por una sonda magnetica. Permitenos detectar directamente lo que buscamos y ya ha sido testado para el factor de necrosís tumoral $\alpha$. (10).

\subsection{INDICADORES DE LA RESPUESTA INFLAMATORIA}

La respuesta inflamatoria en la EP es resultado de la presencia de uno o varios patógenos y/o de la respuesta de los tejidos que la sufren, y que, a nivel bioquímico se traduce en la liberación de un conjunto de mediadores resultantes de la activación de varios sistemas como es el complemento, la coagulación, la fibrinolisis y las kininas; y a nivel celular en la presencia de determinados tipos celulares que participan en la respuesta defensiva del huésped frente a dicha agressión. La respuesta inflamatoria esta mediada fundamentalmente por neutrófilos que representan más del $50 \%$ de los leucocitos en circulación, y que son identificadas en el surco gingival por la presencia de enzimas extracelulares de origen lisosómico. A continuación se describe en que médida la identificación de la presencia de estos mediadores y de su cantidad 


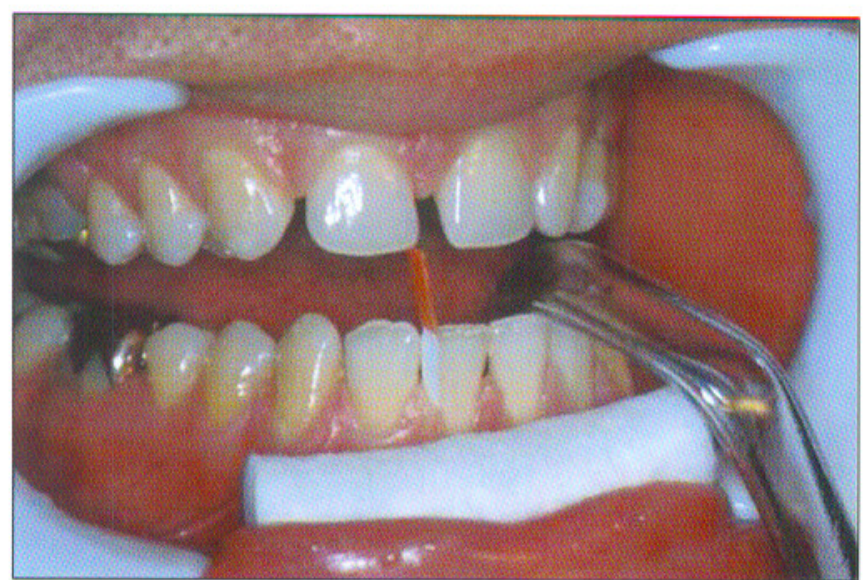

Figura 4.

servirá como diagnóstico e identificación de la severidad de la enfermedad periodontal.

\subsubsection{Prostaglandinas}

La prostaglandina E2 (PgE2) tiene efectos preinflamatórios e imunorreguladores que interfieren con la función y la respuesta celular (11).

Los estudios de Offenbacher et al. 84" (12) evidenciaron que estos mediadores inflamatorios liberados por acción de la ciclooxigenasa sobre el ác. araquidónico están relacionados con la pérdida de inserción. La PgE2 es liberada por los macrófagos y tiene como acción mediar en la respuesta inflamatoria mediante el reclutamiento celular, la producción de colagenasa y la estimulación de la actividad osteoclástica. Se ha especulado con que que la detección de niveles altos de PgE2 en el fluido crevicular podría ser un indicador de pérdida de inserción en los 6 meses siguientes a su aparición.

Por este motivo, la presencia de prostaglandinas podría aparecer en niveles progresivamente mayores en pacientes clínicamente sanos, y en pacientes con gingivitis y periodontitis establecidas. En el momento actual, el único test diagnóstico para la identificación de la PgE2 es el ELISA.

\subsubsection{Elastasa}

La elastasa sintetizada en los tejidos gingivales por los PMN's es detectada, con analisis bioquímico y histoquimico. Actua degradando los proteoglicanos y activando colagenasas latentes. La elastasa esta aumentada en la inflamación gingival, en el aumento de la profundidad de la bolsa y en la perdida del nível de inserción y de hueso, disminuyendo en localizacio- nes sanas como resultado de un tratamiento eficaz (13). Estos estudios demonstraron que está associada a perdida de nivel de inserción cuando se encontra presente a niveles elevados.

\subsubsection{Glucuronidasa}

La glucuronidasa es una enzima que se utiliza como marcador de la liberación de granulos primarios de los neutrófilos. Bajo este principio, la detección de esta enzima se utiliza para determinar la actividad de los linfocitos neutrofilos en el fluido crevicular.

Lamster et al. encontraron una relación positiva entre niveles elevados de esta enzima y la pérdida del nivel de inserción (14). Asimismo, estudios transversales (14) han demuestrado que existe una relación entre estas enzimas y la inflamación gingival, el aumento de la profundidad de sondaje, y la perdida ósea. Incluso se ha asociado el aumento selectivo de algunas enzimas, como la alfa-glucorunidasa con la presencia y actividad de algunas espiroquetas (Porphiromas gingivalis e Prevotella intermédia). De esta forma, podría considerarse como un factor de predictibilidad de futura perdida de inserción, por cuanto la detección de las bacterias sería previa a la destrucción periodontal.

Hasta el momento actual, en la literatura solo existe evidencia de la relación entre el aumento de b-glucuronidada y elastasa con la pérdida de nivel de inserción, y por tanto, con enfermedad activa. Por el contrario, otros indicadores de la actividad de los neutrofilos no tienen aún su relación provada. Por ejemplo, el Leucotrieno B4, resultante de la acción de la lipooxigenasa sobre el Ac. Araquidónico, aunque se encuentre aumentado hasta 4 veces sobre los valores normales en pacientes con periodontitis, sólo nos indica la existencia de enfermedad en ese momento determinado; es decir, carecería de valor predictivo sobre la pérdida de inserción.

\subsubsection{Colagenasa}

Las colagenasas son enzimas que tienen como función degradar el colágeno, y son sintetizadas por macrofagos, fibroblastos, queratinócitos y neutrófilos, tras estimulación por citoquinas y otros produtos bacterianos. En el entorno periodontal, estas enzimas se originan fundamentalmente a partir de los neutrofilos localizados en el surco crevicular. Además, estas células tienen la capacidad de producir los factores inhibidores de las metaloproteinasas, enzimas involucradas igualmente en la destrucción del colágeno. La actividad de la colagenasa presente en el tejido gingival y en el fluido crevicular parece estar aumentada cuan- 
do existe gingivitis, como se ha evidenciado experimentalmente a través de modelos animales (15) y su concentración estaría relacionada con la inflamación y la gravedad de la enfermedad. A través de diversos estudios se ha intentado relacionar la presencia de colagenasa con la enfermedad periodontal, encontrándose una correlación positiva tanto para la periodontitis del adulto como, para la periodontitis juvenil localizada, en relación a los niveles detectados en individuos sanos (16). No obstante, otros autores han referido la inexistencia de una relación constante entre ambas, de forma que los niveles de colagenasa se pueden encontrar fluctuando durante las diferentes fases del tratamiento. Por este motivo, la determinación de altas concentraciones en algunas fases pueden no corresponder a periodos de actividad periodontal o de pérdida de inserción.

Tomando como objeto de estudio la rebsorción ósea, tampoco ha sido posible establecer una correlación con los niveles de colagenasa (21). Posiblemente, esta diversidad en los resultados tiene su origen en el hecho de que esta enzima se puede osriginar en diversos puntos del entorno periodontal, no sólo por los neutrófilos del surco crevicular.

Desde el punto de vista técnico, el principal problema a la hora de determinar los niveles de colagenasa en el fluido crevicular es el tiempo que requiere el procesado de los tests. Mancini et al. (17) han desarrollado un método para cuantificar los niveles de colágenasa, conocido como análisis de colagenasa con biotina soluble (SBA). Este método está basado en la quimioluminiscencia, y permite detectar lo productos de destrución de la biotina por la colagenasa. Según los autores, este es un método de gran especificidad, simple y rápido, y que permite distinguir entre los estados de salud y actividad periodontal.

A pesar de las expectativas creadas, no conviene olvidar que hasta la fecha, el único estudio longitudinal existente no ha permitido probar una relación entre el aumento de las colagenasas y la perdida de inserción.

\subsubsection{Fosfatasa alcalina y fosfatasa ácida}

La fosfatasa alcalina es una enzima segregada por células de estirpe mesenquimal, y linfocitos neutrófilos, pero que también puede tener su origen en algunas bacterias. La presencia de fosfatasa alcalina está relacionada con el metabolismo óseo, siendo detectada a nível de los PMN. Al revés, la fosfatasa acida presente en las células del fluido crevicular no está asociada con cualqiuer parâmetro de actividad o gravedad de enfermedad.

La relación entre la fosfatasas ácida y alcalina y aquellos procesos en los que existe reabsorción ósea ha justificado su estudio como marcadores en la enfermedad periodontal (18). Chapple et al. (19 ) han considerado la posiblidad de utilizar la fosfatasa alcalina en el fluido crevicular como predictor de la pérdida de inserción en pacientes con periodontitis del adulto, mediante quimioflurescencia. Sin embargo, los resultados obtenidos solo permiten sugerir una débil relación entre el aumento de la fosfatasa alcalina y la pérdida de inserción.

\subsubsection{Proteinasas}

Las proteínasas como la catepsina B y L son enzimas involucradas en la degradación de componentes extracelulares como el colageneo, participando también activamente en la reabsorción ósea. En relación con la enfermedad periodontal se ha sugerido que las catepsinas pueden estar asociadas al aumento de la inflamación gingival, profundidad de sondaje, nível de inserción y perdida ósea $(20,21)$. La catepsina B parece ser un marcador fiable de la pérdida progresiva de inserción. De esta forma, su concentración aparecería disminuída en localizaciones sanas, y en zonas afectadas después del tratamiento periodontal. Otra catepsina, la D, se encuentra aumentada aquellas situaciones que comportan inflamación gingival, y por tanto aumento de la profundidad de sondaje, pérdida de inserción y reabsorción ósea. No obstante, y al igual que con otros marcadores, todavía no existe ningún estudio longitudinal que relacione claramente el aumento de esta proteasa con la enfermedad periodontal.

\subsubsection{Tripsina}

La tripsina es una enzima presente en grandes cantidades en los tejidos gingivales y en pequeña cantidad en el fluido crevicular, y que tiene la capacidad de separar la fracción tercera del sistema de complemento y activar la colagenasa en estadio latente. Aumenta con la perdida de inserción y la reabsorción ósea, y disminuye despúes del tratamiento periodontal (2l).

\subsubsection{Dipeptidilpeptidasa}

La DPPII es una enzima lisosomica presente en los fibroblastos y tambien en los macrofagos a nível del fluido crevicular. La DPPIV está presente en los lisosomas de los macrofagos, fibroblastos y linfocitos T. La DPPII es activa en medio ácido, mientras que DPPIV es activa en medio alcalino, estando ambas presentes en los tejidos gingivales y en el fluido crevicular. Son diferentes de las DPP bacterianas porque están relacionadas directamente con la gravedad de la enfer- 
medad. En las localizaciones sanas y en las localizaciones afectadas despúes del tratamiento periodontal eficaz tienen un valor próximo a cero. Por esta razón se han propuesto como posibles marcadores de una futura pérdida de inserción.

\subsubsection{Calproteina}

Es una proteina leucocitaria, asociada a diversas enfermedades de naturaleza inflamatoria, como la Atritis Reumatoide. Puede encontrarse en el fluido crevicular, estando en concentraciones aumentadas en las bolsas periodontales si se compara con surcos gingivales sanos. Los estudios de Kido Jun-ichi et al. (22) analizando el nivel de calprotectina, PgE2, e Illb mediante ELISA, evidenciaron una correlación positiva entre sus concentraciones y los indicadores clínicos de profundidad de sondaje e indice de sangrado. Los mismos estudios indican que los niveles de calproteina esta aumentados cuando el indice de sangrado es mayor. Otro estudio, verificó la existencia de una relación entre sus niveles y los de otros marcadores bioquímicos como la colagenasa o la aminotransferasa-aspartato, sugeriendo que en un futuro podrían ser útiles como marcadores de la inflamación periodontal (23).

\subsection{TESTS DE DIAGNÓSTICO BASADOS EN LOS NIVELES DE ENZIMAS PROTEOLÍTICAS E HIDROLÍTICAS EN EL FLUIDO CREVICULAR}

Se han comercializado dos tests para detectar estas enzimas en fluido crevicular y así evaluar y preveer una futura pérdida de inserción, que se conocen como Periocheck ${ }^{\circledR}$ y Prognostik ${ }^{\circledR}$.

\subsubsection{Periocheck ${ }^{\circledR}$ ( ACTech )}

El Periocheck ${ }^{\circledR}$ consiste en una tira de papel que introducida en el surco periodontal permite obtener una muestra de fluido crevicular, y, a partir de ella, determinar la presencia de proteinasas neutras en el fluido crevicular, como la colagenasa. Tras su introducción en el surco, la tira se coloca en contacto con un gel de colágeno, que, una vez incubado a $43^{\circ} \mathrm{C}$, evidencia una alteración de color que depende de la cantidad de enzima presente. La intensidad del color es evaluada mediante una escala de 0 a 2 , que es comparada con un índice de referencia colorímetrico estándar (muestra tres gamas de color), incluidas en el propio test.

\subsubsection{Prognostik ${ }^{\circledR}$ ( Dentsply )}

Este test permite detectar la presencia de serina y elastasa en el fluido crevicular. La recogida de la muestra se realiza de la misma forma que en el test anterior, pero en este caso, la tira contiene un péptido reactivo que permite detectar la presencia de elastasa. La elastasa será evidenciada mediante la adición de un substracto marcado con un grupo fluorescente, conocido como AFC. La reacción se produce tras un periodo de 4 a 8 minutos, momento desde el cual es posible detectar la emisión de fluorescencia mediante luz ultravioleta. La comparación con una escala de referencia permite cuantificar el resultado.

Los tests de diagnóstico además de ser capaces de detectar la presencia de algún marcador asociado a la actividad de la enfermedad periodontal, deben reunir algunas características, como ser fáciles de utilizar, como ocurre con los métodos colorimétricos, y la de poder ser presentado al paciente. No obstante, al día de hoy estos métodos diagnósticos presentan algunas desventajas, como son:

- la dificuldad para determinar en cada caso el biomarcador más apropiado a la luz de los conocimentos actuales.

- la dificuldad de eligir las localizaciones periodontales y el momento más apropiado para la recojida de la muestra.

- algunos marcadores no son específicos de la inflamación periodontal, y aún en presencia de inflamación, de pérdida de inserción. Así, por ejemplo, un marcador podría tener valor diagnóstico para la presencia de inflamación, y no necesariamente predecir la futura pérdida de inserción.

- en general su coste es elevado (21).

En resumen, del análisis de los estudios anteriores, es posible agrupar los marcadores en dos grandes grupos:

A - Catepsina B, elastasa, DPPII, DPPIV y beta-glicuronidasa, que, en estudios transversales parecen tener valor predictivo de la progresión de la enfermedad periodontal.

B - Colagenasa, tripsina, fosfatasa alcalina, arilsulfatasa y mieloperoxidasa, que se asociarían, según estudios transversales y longitudinales no solo con la actividad, sino con la intensidad de la enfermedad (21).

\subsection{INDICADORES DE LA RESPUESTA INMUNE CELULAR EN EL FLUIDO CREVICULAR}

A partir de los años 70 comienza el interés por conocer el papel de los linfocitos $\mathrm{T}$ en la enfermedad 
periodontal. El estudio de la inmunidad celular y su posible implicación en los mecanismos etiopatogénicos periodontales presentaba dos importantes escollos. Por un lado, la gran cantidad de mediadores identificables en el surco gingival, y por otro, la dificultad para su detección cuando se encontraban en pequeñas concentraciones. La identificación de un grupo particular de mediadores caracteristicos de los linfocitos $\mathrm{T}$ y de los macrofagos, conocidos como citoquinas, junto con el desarollo de anticuerpos monoclonales para dichas moleculas permitieron un importante avance en este campo.

Son péptidos sintetizados por linfocitos y macrófagos capaces de unirse a un receptor específico de la membrana de otra célula. Existen varios tipos con funciones diferentes, y con repercusión clínica también diferente.

\section{1) Interleuquina 1}

Existen dos formas de interleukina 1, la $\alpha$ y $\beta$, que pueden unirse a un mismo receptor de la membrana. Ambas formas comparten sólo el $25 \%$ de la secuencia de aminoácidos, estando asociadas a estadios iniciales de la inflamación, que incluyen: modificación de células epiteliales, estimulación de la producción de proteinasas y de prostoglandina E2, así como activación de osteoclastos. Los estudios publicados hasta la fecha no permiten establecer una relación directa entre estas proteinas y las diversas formas de periodontitis (24).

La Ill está presente en los tejidos gingivales inflamados y en los casos de periodontitis, encontrándose en concentraciones menores en localizaciones sanas. No se ha establecido relación con la profundidad de sondaje, aunque parece que su concentración tiende a disminuir despúes del raspado y alisado radicular.

\section{2) Factor de necrosis tumoral $\alpha$}

El factor de necrosis tumoral a es secretado por macrófagos en respuesta a diversos estímulos como son la presencia de supuestos periodontopatógenos (24). Actúa como activador de la reabsorción ósea y de la liberación de colagenasa por los fibroblastos. Los estudios de Rossomando et al. (25), relativos al estudio de localizaciones con diferente grado de enfermedad periodontal no permitieron encontrar relación entre el TNF $\alpha$ en el fluido crevicular y ningún parámetro clínico de los evaluados (índice gingival, indice de placa y profundidad de sondaje).

\begin{tabular}{|llll|}
\hline IFNg & Fuente celular & Célula diana & Acción \\
\hline IL1 & Cél. NK & $\begin{array}{l}\text { Linfocitos } \\
\text { Monocitos } \\
\text { Cél. epiteliales }\end{array}$ & $\begin{array}{l}\text { Inmunorregulación } \\
\text { Diferenciación B } \\
\text { Antiviral }\end{array}$ \\
\hline IL2 & $\begin{array}{l}\text { Monocitos, Cél. B } \\
\text { Macrófagos, } \\
\text { Fibroblastos, } \\
\text { Cél. endoteliales }\end{array}$ & $\begin{array}{l}\text { Timocitos } \\
\text { Neutrófilos } \\
\text { Cél. B } \text { Y T }\end{array}$ & $\begin{array}{l}\text { Inmunorregulación } \\
\text { Inflamación } \\
\text { Fiebre }\end{array}$ \\
\hline IL3 & Cél. NK & $\begin{array}{l}\text { Cél. B y T } \\
\text { Monocitos } \\
\text { Macrófagos }\end{array}$ & $\begin{array}{l}\text { Proliferación } \\
\text { Activación }\end{array}$ \\
\hline IL4 & Cél. T & Stem cels & Dolor \\
IL5 & Cél. T & Cél. B y T & División y diferenciación \\
\hline IL8 & Macrófago & Granulocitos & Destrucción ósea \\
\hline TNF $\alpha$ & Macrófago & Fibroblastos \\
TNF $\beta$ & Cinfocitos & Cél. endoteliales & Quimiotaxis \\
\hline
\end{tabular}

Tabla 1. Acción de las citoquinas en la respuesta celular. 


\section{3) Interlequina 6}

Es una proteína producida por macrófagos, linfocitos B y T, fibroblastos y células endoteliales. Es responsable de una gran variedad de funciones, tales como la estimulación de la produción de proteínas de la fase aguda de la inflamación, la diferenciación de linfocitos B y la reabsorción ósea (26). La Il6 se puede identificar en el fluido crevicular, pero no ha sido posible aún establecer una relación entre el aumento de su concentración y la enfermedad periodontal $(27,28)$. La Il6 puede estar aumentada en las periodontitis refractarias (29) y junto con la Il2 puede tener alguna importancia en la predictibilidad de la perdida de nivel de inserción.

\section{4) Interleuquina 8 (118)}

La Il8 es un factor quimiotáctico para los neutrófilos y puede ser detectado en el fluido gingival. Su concentración se asocia con el nivel de Il-1 $\beta$ (30).

\section{CONCLUSIÓN}

El diagnóstico de la periodontitis, como se hizo referencia al inicio de esta revisión, solo permite, en el momento actual, detectar aquellas localizaciones en las que se ha producido pérdida de inserción. No disponemos de ningún método diagnóstico que nos permita realizar un detección precoz de aquellas localizaciones que vayan a experimentar futura pérdida de inserción. La investigación de nuevas técnicas basadas en el análisis de determinados marcadores, como hemos reseñado a lo largo de esta revisión, abre un nuevo campo en el diagnóstico periodontal. Douglass y Fox et al. (31) sugirieron que los test diagnósticos utilizados para identificar la actividad de enfermedad podrían ser útiles para algunos pacientes, como aquellos en fase de mantenimento o con periodontitis refractaria. Sin embargo, la utilidad clínica de estos test es aún limitada, por cuanto no existe todavía una forma clara de diagnosticar la enfermedad periodontal de forma prospectiva.

\section{SUIMIMARY}

The Tradicional Methods of Diagnosis aren't enought predictible, and they only give us na retrospective diagnosis about the attachement level. So, with the objective of improving the predictibility of our diagnosis, new methods are desenvolved to detecting the progression of periodontal disease, based on inmunological response.

Nowadays the clinicians have to know this new methods, to understand when they are realy useful.

It has been done un bibliographic revision of the 2 last years in the Journal Clinical of Periodontology y en Journal of Periodontol.

\section{CORRESPONDENCIA:}

Faria R.

Master de Periodoncia de la UCM

Plaza Ramón y Cajal, s/n

28040 Madrid

\section{BIBLIOGRAFÍA}

1. Zambon,JJ.; Haraszthy,VI. (1995). "The laboratory diagnosis of periodontal infections." Periodontology $2000 ; 7,69-82$.

2. Paquette,DW; Williams,RC. (1994). "Rapid chairside diagnostic tests for periodontitis: current status and critical assessment." Periodontology 2000;15, 4-8.

3. Russell,et al. (1967). "Epidemiology of periodontal disease." International Dental Journal; 17, 282-296.

4. Loe,H.; Anerud,A.; Boysen,H.; Morrison,E. (1986). "Natural history of periodontitis disease in man. Rapid moderate and loss of attachement in Sri Lankan laborers 14 to 46 years of age." J. Clinical Periodontology; $13,431-440$.

5. Haffajee,AD.; Socransky,SS. (1994). "Microbial etiology agents in destructive periodontol diseases." Peiodontology 2000; 5, 78-111.

6. Cimasoni (1983) "Crevicular fluid update", in Myers HM. Ed. Monografs in Oral Science.

7. Bickel M., Cimasoni G. (1984) "Reliability of volume measurements with the new Periotrom 6000." J Periodont Res.; 19, 313-316.

8. Hinrichs JE., Bandt CL., Smith JÁ. (1984) "Relative error (variability) associated with na improved instrument for measuring gingival crevicular fluid." J. Periodontol; 55: 294-298. 
9. Attstrom R. (1970) "Presence of leukocytes in crevices of heathy and chronically inflamed gingivae." J. Periodont Res.; 5, 42-47.

11. Goodson JM., Dewhirst FE., Brunetti A.(1974) " PgE2 levels and human periodontal disease." Prostoglandins; 6: 81-85.

12. Offenbacher S., Collins JG., Arnold RR. (1984) "New clinical diagnosis strategics based on patogenic disease." J Period. Res.; 28: 523-535.

13. Palcanis Kg., Larjava IK., Wells BR. (1992) "Elastase na indicator of periodontal disease progression." J. Periodontol; 63: 237-242. 18) Binder TA., Goodson JM. Socransky SS. (1987) "Gingival fluid levels of acid and alkaline phosphatase." J. Periodont rest.; 22: 14-19.

14. Lamster IB., Holmes LG., Williams KB. (1995) "The relationship of beta-glicuronidase activity in crevicular fluid to probing attachement loss in patients with adult periodontitis." Journal of Clinical Periodontology.

15. Kryshtalskyj,E. et al (1986) "Correlation of collagenolytic enzymes and inhibitors in gingival crevicular fluid with clinical and microscopic changes in experimental periodontitis in the dog." Archives of Oral Biology 31, 21-31.

16. Lee W., Aitken S., Kulkarni G. (1991) "Collagenase activity in recurrent periodontitis: relationship to disease progression and doxycycline therapy." Journal Periodont Rest.; 26: 479-485.

17. Mancini S., Romanelli R., Laschinger C.,Overall C. (1999) "Assessment of a novel screening test for neutrophil collagenase activity in the diagnosis of periodontal disease."J. Periodontol; 70: 1292-1302.

19. Chapple ILC., Garner I., Saxby MS., (1999) "Prediction and diagnosis of attachement loss by enhanced chemiluminescent assay of crevicular fluid alkaline phosphatase levels." Journal of Clinical Periodontology; 26: 190198.

20. Lah,T. et al (1996) "Detection of cathepsin L-like proteinase and cathepsin D in gingival fluid." Journal of Periodontal Research 21,504-509.
21. Eley,BM. et al (1992) "Cathepsin B/L,elastase-, tryptase, trypsin-, and dipeptidyl peptidase IV-like activities in gingival crevicular fluid: Correlation with clinical parameters in untreated chronic periodontitis patients." Journal of Periodontal Research, 27, 62-69.

23. Nakamura T., Kido J., Kido R., Ohishi k. (2000) "The association of calprotectin level in gingival crevicular fluid with gingival index and the activitties of colagenase adult periodontitis patients." J. Periodontol; 71:361-367.

24. Kido J:, Nakamura T., Kido R., Ohishi k. (1999) "Calprotectina in gingival crevicular fluid correlates with clinical and biochemicalmarkers of periodontal diesease."J. Clinical Periodontology; 26: 653-657.

24. Lindermann RA., Economon JS. (1988) "Prodution of interleukine-1 and tumor necrosis factor by human peripheral monocytes activated by periodontal bacterias and extracted lipopolysaccharides." J. Dent. Res.;68: 1131-1135.

25. Rossomando Ef., White LB., Hadjimichael J. (1992) "Immunomagnetic separation of tumor necrosis factor alfa." J. Chromatografy ;583: 19-26.

26. Takahashi K., Takashiba S., Nagai A. (1994) "Assesement of interleukine-6 in the pathogenisis of periodontal disease." J. Periodontol 1994: 27: 511-521.

27. Geivelis M., Turner DW., Pederson ED: (1990) "A sensitive ELISA-based assay for interleukin-6." J. Dent.Res.; 69.

28. Rowland RW:, Mestecky J., gunssoly JC. (1993) "Serum IgG and IgM levels to bacterial antigens in necrotizing ulcerative gingivitis." J. Periodontol; 64:195-201.

29. Reinhardt,RA. et al (1993) "Gingival fluid IL-1 and IL-6 levels in refractory periodontitis." Journal of Clinical Periodontology; 20,225-231.

30. Payne JB., Reinhardt RA, Masada MP. (1993) "Gingival crevicular fluid I18." J.Periodont. Rest. ;28: 451-453.

31. Douglass CW:, Fox CH:, (1991) "Determining the value of periodontal diagnostic test." J. Periodontol;62: 721730. 